\title{
DEFORMATIONS, RELATED DEFORMATIONS AND A UNIVERSAL SUBFAMILY
}

\author{
BY \\ KIMYONG KIM(1)
}

Introduction. Let $n \geqq 2,1=d_{1}, d_{2}, \cdots, d_{n}$ be integers such that $d_{i} \mid d_{i+1}$ and

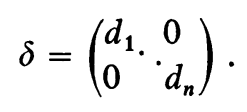

Then, for each element $z$ of the Siegel's generalized upper-half plane $H_{n}$, $(n \times 2 n)$-matrix $(z, \delta)$ is a Riemann matrix of an abelian torus $T(z)$ which admits

$$
P=\left(\begin{array}{ll}
0 & \delta \\
-\delta & 0
\end{array}\right)
$$

as a principal matrix. By means of theta functions, $P$ gives a polarization on $T(z)$ and we denote by $(T(z), P)$ the resulting polarized abelian torus.

For $z$ and $z^{\prime}$ in $H_{n},(T(z), P)$ and $\left(T\left(z^{\prime}\right), P\right)$ are isomorphic if and only if there are complex $(n \times n)$-matrix $C$ and $(2 n \times 2 n)$-integer matrix $M$ such that $(z, \delta) M=C\left(z^{\prime}, \delta\right)$ with ${ }^{t} M P M=P$. On the other hand, $\mathrm{Sp}(n, R)$ operates on $H_{n}$ by $z \rightarrow(A z+B)(C z+D)^{-1}$, where

$$
\left(\begin{array}{ll}
A & B \\
C & D
\end{array}\right)
$$

is an element of $\operatorname{Sp}(n, R)$. And for a fixed $\delta$, there is a discrete subgroup $\Gamma(\delta)$ of $\operatorname{Sp}(n, R)$ such that the $\Gamma(\delta)$-orbit of $z$ in $H_{n}$ coincides with the polarized isomorphism class of $(T(z), P)$ when we identify $z \in H_{n}$ with $(T(z), P)$. We may regard $H_{n} / \Gamma(\delta)$ as a variety of moduli for the set of polarized abelian tori arising from $H_{n}$.

By means of theta series, which depend on an integer $\mu \geqq 3$, we can embed (as complex analytic manifolds) all $T(z), z \in H_{n}$, simultaneously into a projective space $\boldsymbol{P}^{N}$ of dimension $\mu^{n}|\delta|-1$. Let $\mathscr{U}(\delta, \mu)$ be the set of abelian varieties in $\boldsymbol{P}^{N}$ with the natural polarizations (i.e., the polarization determined by hyperplane

Received by the editors February 22, 1965.

(1) The content of this paper forms my doctoral dissertation, Brandeis University, May 1964. I would like to express my gratitude here, especially to Professor T. Matsusaka, who has guided me in this work. 
sections) which are images of the above embedding or their projective transforms. Then, the set of Chow-points of the members of $\mathscr{U}(\delta, \mu)$ form a $Q$-open variety and the polarized isomorphism classes of the members of $\mathscr{U}(\delta, \mu)$ ? coincide with projective families.

If we regard a construction of variety of moduli as finding a locally closed set whose points represent isomorphism classes, then we may enlarge the set $\mathscr{U}(\delta, \mu)$ which depended on $\mu$, to the set $\mathscr{A}(\delta)$ of all polarized abelian varieties which are isomorphic to some member of $\mathscr{U}(\delta, \mu)$. Then we can give another characterization of $\mathscr{A}(\delta)$ : let us call a polarized abelian variety a "deformation" of another if there is a finite chain of polarized abelian varieties connecting the two so that each consecutive one is either isomorphic or one of the pair is a specialization of the other over the field $Q$. Then, for $A \in \mathscr{A}(\delta)$, let $\Sigma(A)$ be the set of deformations of $A$. We have $\mathscr{A}(\delta)=\Sigma(A)$ [10], [4].

By rearranging the above sequence of observations backwards, we have the following set which becomes susceptible of generalization to other polarized varieties. Let $A_{0}$ be a polarized abelian variety. Then, there is a subset $\mathscr{U} \subset \Sigma\left(A_{0}\right)$ such that (1) for any $A \in \Sigma\left(A_{0}\right)$ there is $U \in \mathscr{U}$ with $\boldsymbol{A} \cong \boldsymbol{U}$, (2) underlying varieties of the members of $\mathscr{U}$ are in a fixed projective space, and (3) the set of Chow-points of the underlying varieties of the members of $\mathscr{U}$ is a $Q$-open variety. We may call such $\mathscr{U}$ a universal subfamily of $\Sigma\left(A_{0}\right)$ and there are many such subfamilies for $\Sigma\left(A_{0}\right)$. The special one we have considered, $\mathscr{U}(\delta, \mu)$, renders fibering by projective families and leads to a construction of a variety of moduli for $\Sigma\left(A_{0}\right)$. In this sense, $\mathscr{U}$ may be considered as a geometric counterpart to $H_{n}$ which was an intermediate stage toward $\cdots H_{n} / \Gamma(\delta)$.

In the case of nonsingular curves of fixed genus $n$, Baily constructed a variety of moduli by means of their Jacobians and $H_{n} / \Gamma\left(I_{n}\right)$, where $I_{n}$ is the identity $(n \times n)$-matrix. From our point of view, the following aspects of the curves are of main interest:

(1) the canonical map $\phi: C \rightarrow J$ of a nonsingular curve $C$ into its Jacobian $J$ is biregular (birational) and the image $\phi(C)$ determines a polar divisor $\Theta$ with $l(\Theta)=1$, i.e., $(J, \widetilde{\Theta}) \in \mathscr{A}\left(I_{n}\right)$ if we denote the polarization determined by $\Theta$ by $\widetilde{\Theta}$,

(2) the canonical map $\phi: C \rightarrow J$ is compatible with the specialization of the curve $C$ (we consider only nonsingular specializations),

(3) Hoyt's result [3] that the set of Jacobians of curves of fixed genus $n$ in a family of polarized abelian varieties (here we may take $\mathscr{U}\left(I_{n}, \mu\right)$ ) forms a locally closed family.

When we have a variety of moduli as in the case of curves, it seems idle to look for a universall subfamily. But the case of curves suggests a tentative step of generalization to higher dimensions. In this paper, we will consider some additional structure on a nonsingular polarized variety and one restrictive enough 
notion of "deformation" so that the set of deformation admits a universal subfamily.

Let $\boldsymbol{V}$ be a polarized variety and suppose that the underlying variety $V$ of $\boldsymbol{V}$ has following properties: (1) the dimension of the image of $V$ by an albanese map of $V$ is the same as that of $V$, and (2) no curve on $V$ is crushed to a point by the albanese map. If (1) and (2) are satisfied, we will call $V$ an " $\alpha$-variety" and define $\alpha$-deformations of $\boldsymbol{V}$. In $\S 2$, we will construct a universal subfamily for the set of $\alpha$-deformations, while in $\S 1$ we will develop terminology in a slightly more general setting, the main guideline being Matsusaka-Mumford [9].

1. Related deformations. Notational CONVENTION. While our basic terminology will be that of [11], we will also follow definitions in [12], [8] and [9]. As specific assumptions and conventions of this paper, we take the field of rational numbers, $\boldsymbol{Q}$, as our ground field and confine ourselves to projective geometry. For example, by a specialization of a variety, denoted by $\sim \rightarrow$, we will mean a specialization as a cycle in an ambient projective space defined over $\boldsymbol{Q}$. Furthermore, we will consider polarization only on a nonsingular variety (projective, absolutely irreducible). To avoid repetition, we will denote by "variety $V$ " with boldface the following: a nonsingular variety $V$, called the underlying variety of $V$, has a specific polarization structure $\mathscr{X}$ on it and the pair of concepts $(V, \mathscr{X})$ is denoted by $\boldsymbol{V}$. For example, $\boldsymbol{V} \sim \rightarrow V^{\prime}$ means "on $V$ and $V^{\prime}$ polarizations $\mathscr{X}$ and $\mathscr{X}^{\prime}$ respectively are given and for a polar divisor $X \in \mathscr{X},(V, X) n \rightarrow\left(V^{\prime}, X^{\prime}\right)$ over $V \backsim \rightarrow V^{\prime}$ (over $Q$ ) with $X^{\prime} \in \mathscr{X}^{\prime}$." As in the above example, if not otherwise mentioned, a capital letter $U$, corresponding to a boldfaced $U$ which represents a polarized variety, will be understood as denoting the underlying variety of $\boldsymbol{U}$.

Definition 1. A morphism (everywhere defined birational map) $\phi: V \rightarrow V^{\prime}$ of the underlying variety $V$ of $V$ into that of $V^{\prime}$ will be called a (polarized) morphism of $\boldsymbol{V}$ into $\boldsymbol{V}^{\prime}$, written again by $\phi: V \rightarrow V^{\prime}$, if there is a polar divisor $X^{\prime}$ in $\mathscr{X}^{\prime}$ such that $\phi^{-1}\left(X^{\prime}\right)=\operatorname{pr}_{1}\left[\Gamma_{\phi} \cdot\left(V \times X^{\prime}\right)\right]$ is defined and is in $\mathscr{X}$.

This definition does not depend on the choice of a polar divisor $X^{\prime}$ in $\mathscr{X}^{\prime}$. By the result in [7] of a regular maximal algebraic family of positive divisors on a nonsingular variety, it is enough to check for the case $X^{\prime} \sim Y^{\prime}$ (linear equivalence on $\left.V^{\prime}\right)$ with both $\phi^{-1}\left(X^{\prime}\right)$ and $\phi^{-1}\left(Y^{\prime}\right)$ being defined. Then, by the Corollary to Theorem 14, Chapter VIII of [11], we have $\phi^{-1}\left(X^{\prime}\right) \sim \phi^{-1}\left(Y^{\prime}\right)$ on $V$. We also notice $\left({ }^{2}\right)$ that $\phi$ has to satisfy the following: for any point $a \in V^{\prime}$, the point set $\phi^{-1}(a)$ has to be finite. $V^{\prime}$ being polarized and $\phi$ being into, we may consider the case where $V^{\prime}$ is a projective space with the natural polarization. Let $L(\phi)$ be the linear system defining $\phi: V^{n} \rightarrow P^{N}$ (hence $L(\phi) \subset \mathscr{X}$ ) and let $m=\operatorname{dim} \phi(V)$. Suppose $a \in \boldsymbol{P}^{N}$ and the set $\phi^{-1}(a)$ is not finite. Let $W^{s}$ be a component with maximal dimension in $\phi^{-1}(a)$ with $s \geqq 1$. Then, $m-(n-s) \geqq 0$,

(2) This fact was pointed out to me by the referee, and this eliminates many of the vacuous concepts I have originally dealt with. 
and we can choose general hyperplanes $H_{1}, H_{2}, \cdots, H_{n-s}$ in $\boldsymbol{P}^{N}$ such that $a \notin H_{i}$, $\phi^{-1}(V) \cdot H_{1} \cdots H_{n-s} \neq \varnothing$ is defined and $\phi^{-1}\left(H_{i}\right)$ are also defined. Going to numerical equivalence class, $W^{s} \cdot X^{(n-s)}=0$ with $X \in \mathscr{X}$. This contradicts Nakai's criterion of nondegeneracy [6] of $X$, namely there exists al positive s-cycle $W^{s}$ such that $W^{s} \cdot X^{(n-s)}$ is not positive.

Definition 2. Let $\boldsymbol{V}$ and $\boldsymbol{V}^{\prime}$ be varieties. They are said to be immediate deformations of each other if $V$ is isomorphic to $V^{\prime}$ or one of them is a specialization of the other. We say that they are deformations of each other if there are a finite number of varieties $V=V_{0}, V_{1}, \cdots, V_{s}=V^{\prime}$ such that each consecutive one is an immediate deformation of the other. By $\Sigma(V)$, we denote the set of deformations of variety $\boldsymbol{V}$.

Definition 3. When $\Sigma\left(U_{0}\right)$ is given, a variety $V$ is said to be $\Sigma\left(U_{0}\right)$-related if there is a morphism $\phi ; V \rightarrow U$ for some $U$ in $\Sigma\left(U_{0}\right)$. When this is so, we denote by $(\boldsymbol{V}, \phi)$ the $\Sigma\left(\boldsymbol{U}_{0}\right)$-related variety $\boldsymbol{V}$ with relation $\phi$.

Definition 4. Let $\Sigma=\Sigma\left(U_{0}\right)$ be given, and $(\boldsymbol{V}, \phi)$ and $\left(\boldsymbol{V}^{\prime}, \phi^{\prime}\right)$ be $\Sigma$-related varieties. They are said to be isomorphic to each other (with $\Sigma$-relation), written $(\boldsymbol{V}, \phi) \cong\left(\boldsymbol{V}^{\prime}, \phi^{\prime}\right)$, if (a) there is an isomorphism $\alpha ; \boldsymbol{V} \rightarrow \boldsymbol{V}^{\prime}$ and (b) there is an isomorphism $\beta: U \rightarrow U^{\prime}$ such that $\beta 0 \phi=\phi^{\prime} 0 \alpha$, where $\phi: V \rightarrow U$ and $\phi^{\prime}$ : $V^{\prime} \rightarrow U^{\prime}$. Similarly, $\left(V^{\prime}, \phi^{\prime}\right)$ is said to be a $\Sigma$-related specialization of $(\boldsymbol{V}, \phi)$, written $(V, \phi) \sim \rightarrow\left(V^{\prime}, \phi^{\prime}\right)$, if (a) $\boldsymbol{V} \sim \rightarrow V^{\prime}$ and (b) there are $\Sigma$-related $(\boldsymbol{V}, \bar{\phi})$ and $\left(\boldsymbol{V}^{\prime}, \overline{\phi^{\prime}}\right)$ such that $(\boldsymbol{V}, \phi) \cong(\boldsymbol{V}, \bar{\phi}), \quad\left(\boldsymbol{V}^{\prime}, \phi^{\prime}\right) \cong\left(\boldsymbol{V}^{\prime}, \overline{\phi^{\prime}}\right)$ and $\left(\boldsymbol{V}, \bar{U}, \Gamma_{\bar{\phi}}\right)$ $n \rightarrow\left(V^{\prime}, \bar{U}^{\prime}, \Gamma_{\bar{\phi}}\right)$, where $\Gamma_{\phi}$ denotes the graph of $\phi$.

In Definition 2, we can replace "isomorphism", "specialization" and "variety $\boldsymbol{V}_{i}$ ' 'by $\Sigma$-related ones respectively and obtain a notion of $\Sigma$-related deformation. We denote by $\Sigma(V, \phi)$ the set of $\Sigma$-related deformations of $(\boldsymbol{V}, \phi)$, when $\Sigma=\Sigma\left(U_{0}\right)$ is fixed.

In the following, we will restrict ourselves in the type of $\Sigma$, so that the resulting $\Sigma$-related deformation will be seen to be a slight generalization of " $\alpha$-deformation" mentioned in the Introduction.

Definition 5. The set $\Sigma\left(\boldsymbol{U}_{0}\right)$ of deformations of a variety $\boldsymbol{U}_{0}$ will be called "of type $c$ " if there is a positive integer $c$, such that:

(1) for every $U$ in $\Sigma$ and for any basic polar divisor $B$ of $U$, there is a positive $U$-divisor $Z$ which is algebraically equivalent to $c B$, and every such $Z$ is ample;

(2) for every $U$ in $\Sigma$, the set of positive $U$-divisors $Z$, which are algebraically equivalent to $c B$ for some basic polar divisor $B$ of $\boldsymbol{U}$, form an irreducible algebraic family $\mathscr{F}(c, \boldsymbol{U})$, and $l_{u}(Z)$ depends only on $\Sigma$. $\left(l_{u}(Z)=\operatorname{dim}|Z|+1\right.$, where $|Z|$ denotes the complete linear system on $U$ determined by $Z$.)

From now on, we fix a set of deformations of a variety $U_{0}, \Sigma$, of type $c$, and a $\Sigma$-related variety $\left(V_{0}, \phi_{0}\right)$ with $\operatorname{dim} V_{0} \geqq 2$. We may rearrange the notations so that $\phi_{0}: V_{0} \rightarrow U_{0}$.

For any $(\boldsymbol{V}, \phi) \in \Sigma\left(V_{0}, \phi_{0}\right)$, and for any $Z \in \mathscr{F}(c, U)$, we can apply Bertini's theorems on the linear system $\phi^{-1}(|Z|$ ) (on $V$ ) and assert that there is a non- 
singular variety $W$ on $V$ as a member of $\phi^{-1}(|Z|)$. $\phi$ induces the morphism $\phi_{w}$ : $W \rightarrow U$ and $\operatorname{Tr}_{w}\left(\phi^{-1}(|Z|)\right)=\phi_{w}^{-1}(|Z|)$ on $W$. By the second remark after Definition 1 , members of $\phi_{w}^{-1}(|Z|)$ are nondegenerate $W$-divisors and give an induced polarization on $W$. Then, $\phi_{w}: W \rightarrow U$ is a (polarized) morphism so that $\left(W, \phi_{w}\right)$ is $\Sigma$-related.

With these notations, let's consider the following two sets:

(1) fix a divisor $Z_{0} \in \mathscr{F}\left(c, U_{0}\right)$ and also a nonsingular variety $W_{0} \in \phi^{-1}(|Z|)$, and then we have $\Sigma\left(W_{0}, \phi_{0 w_{0}}\right)$;

(2) $\Sigma^{\prime}$ be the set of all possible pairs $\left(W, \phi_{w}\right)$ with nonsingular variety $W \subset V$, $W \in \phi^{-1}(|Z|)$ while $Z \in \mathscr{F}(c, U)$ and $(\phi, \phi) \in \Sigma\left(V_{0}, \phi_{0}\right)$ vary.

LEMMA 1. $\Sigma^{\prime} \subset \Sigma\left(\boldsymbol{W}_{0}, \phi_{0 w_{0}}\right)$.

As $\left(\boldsymbol{W}_{0}, \phi_{0 w_{0}}\right) \in \Sigma^{\prime}$, it is enough to show that for any $\left(\boldsymbol{W}, \phi_{w}\right) \in \Sigma^{\prime}$ there are a finite number of $\left(\boldsymbol{W}_{i}, \phi_{i w_{i}}\right)(i=0, \cdots, s)$ such that each consecutive one is an immediate deformation of the other in the sense of $\Sigma$-relation, with $\left(W, \phi_{w}\right)$ $=\left(\boldsymbol{W}_{s}, \phi_{s_{3}}\right)$.

Case 1. $\phi=\phi_{0}$ and $Z=Z_{0}$. Taking a sufficiently generic member $\bar{W}$ of $\phi_{0}^{-1}\left(\left|Z_{0}\right|\right)$, we have $\left(W_{0}, \phi_{0 w_{0}}\right) \leftarrow \sim\left(\bar{W}, \phi_{0 \bar{w}}\right) \sim \rightarrow\left(W, \phi_{0 w}\right)$.

Case 2. $\phi=\phi_{0}$ and $Z \in \mathscr{F}\left(c, U_{0}\right)$. By the Case 1 above and property (2) of Definition 5 , it is enough to consider the case where $W_{0}=\phi_{0}^{-1}\left(S_{0}\right), S_{0} \in\left|Z_{0}\right|$ and $\bar{W} .=\phi_{0}^{-1}(\bar{S}), \bar{S} \in|Z|$ so that $\bar{S}$ is a sufficiently generic member of the family $\mathscr{F}\left(c, U_{0}\right)$. Then, $\left(\bar{W}, \phi_{0 \bar{w}}\right) \sim \rightarrow\left(W_{0}, \phi_{0 w_{0}}\right)$.

General case. Over the deformation $\boldsymbol{U}_{0}$ to $\boldsymbol{U}$, members of $\mathscr{F}\left(c, \boldsymbol{U}_{0}\right)$ deform to those of $\mathscr{F}(c, U)$. Hence, in finite steps, this case reduces to Case 1 or 2 above.

With the same notation as above, we obtain a set $\Sigma_{1}\left(V_{0}, W_{0}\right)$ of pairs of varieties $(V, W)$ such that $W \subset V,(V, \phi) \in \Sigma\left(V_{0}, \phi_{0}\right)$ and $\left(W, \phi_{w}\right) \in \Sigma^{\prime}$.

Proposition 1(3). There is a constant $c_{r}$ of the set $\Sigma_{1}$ such that for any integer $m \geqq c_{r}$,

$(r, A) . H^{i}\left(V, \mathscr{L}_{v}\left(m W^{\prime}\right)\right)=0$ for $i \geqq 1$, where $\mathscr{L}_{v}\left(m W^{\prime}\right)$ is the sheaf on $V$ defined by $\left|m W^{\prime}\right|[12]$,

$(r, B)$. The minimal sum of $\phi^{-1}(|Z|)$ and $\left|m W^{\prime}\right|$ on $V$ is complete; where $r=\operatorname{dim} V,(V, W) \in \Sigma_{1}$, and $W^{\prime} \in \phi^{-1}(|Z|)$ such that $W \cdot W^{\prime}$ is defined.

Proof. We will use induction on $r$. Suppose $r>2$ and assume that $(r-1, A)$ and $(r-1, B)$ hold with constant $c_{r-1}$. By Lemma 1, these assumptions hold for the pairs $(W, T)$ in $\Sigma_{1}\left(W_{0}^{r-1}, T_{0}^{r-2}\right)$, which we get from $\Sigma^{\prime}$.

For a given pair $(V, W) \in \Sigma_{1}\left(V_{0}, W_{0}\right)$, and an integer $m>1$, consider the following exact sequence of sheaves determined by respective divisors;

$$
0 \rightarrow \mathscr{L}_{V}\left((m-1) W^{\prime}\right) \rightarrow \mathscr{L}_{V}\left(m W^{\prime}\right) \rightarrow \mathscr{L}_{V}\left(m W \cdot W^{\prime}\right) \rightarrow 0
$$

(3) I am grateful to the referee who has pointed out that this proposition is proved in a much more general context in the forthcoming thesis of S. Kleiman (Ph. D., Harvard, 1965). 
For $m \geqq c_{r-1}$, by $(r-1, A)$, we have an exact sequence

$$
\begin{aligned}
0 & \rightarrow H^{0}\left(V,(m-1) W^{\prime}\right) \rightarrow H^{0}\left(V, m W^{\prime}\right) \rightarrow H^{0}\left(W, m W \cdot W^{\prime}\right) \rightarrow H^{1}\left(V,(m-1) W^{\prime}\right) \\
& \rightarrow H^{\prime}\left(V, m W^{\prime}\right) \rightarrow 0 \rightarrow H^{2}\left(V,(m-1) W^{\prime}\right) \rightarrow H^{2}\left(V, m W^{\prime}\right) \rightarrow 0 \rightarrow \cdots \rightarrow 0 \\
& \rightarrow H^{r}\left(V,(m-1) W^{\prime}\right) \rightarrow H^{r}\left(V, m W^{\prime}\right) \rightarrow 0
\end{aligned}
$$

where we abbreviated $H^{i}\left(V, \mathscr{L}_{V}(D)\right)$ into $H^{i}(V, D)$.

$W^{\prime}$ being a nondegenerate $V$-divisor, a high multiple of $W^{\prime}$ is ample and hence equivalent to a hyperplane section of a variety which is the embedded image of $V$ by the ample linear system. By the semi-fineness of the sheaf determined by a hyperplane section, [12], we get

$$
H^{i}\left(V, m W^{\prime}\right)=0 \text { for } i \geqq 2 \text { and large } m \text {. }
$$

But, for $m \geqq c_{r-1}, H^{i}\left(V,(m-1) W^{\prime}\right)=H^{i}\left(V, m W^{\prime}\right)(i \geqq 2)$ by the exact sequence above. That is, $m$ is large enough if $m \geqq c_{r-1}$ to obtain the above equality.

Let us denote $e_{r, m}=\chi\left(V, m W^{\prime}\right)=\sum_{i=0}^{r}(-1)^{i} h^{i}\left(V, m W^{\prime}\right)$, where $h^{i}\left(V, m W^{\prime}\right)=$ $\operatorname{dim} H^{i}\left(V, m W^{\prime}\right)$. We have $e_{r, m}=h^{0}\left(V, m W^{\prime}\right)-h^{\prime}\left(V, m W^{\prime}\right)$ for $m \geqq c_{r-1} . V$ being nonsingular, $e_{r, m}$ is a specialization invariant of the pair $\left(V, m W^{\prime}\right),[1]$, and hence a constant of the set $\Sigma_{1}\left(V_{0}, W_{0}\right)$ when $m$ is fixed. We also have the result in [9] that $h^{0}\left(V, m W^{\prime}\right) \leqq d_{r, m}$ for a fixed $m$, where $d_{r, m}$ is a constant of the set $\Sigma_{1}\left(V_{0}, W_{0}\right)$. Put $e_{r}=e_{r, c_{r-1}}, d_{r}=d_{r, c_{r-1}}$ and $b_{r}=d_{r}-e_{r}$. Then, for any pair $(V, W)$ in $\Sigma_{1}$, we have $h^{1}\left(V, c_{r-1} W^{\prime}\right) \leqq b_{r}$.

From the exactness of $H^{1}\left(V,(m-1) W^{\prime}\right) \rightarrow H^{1}\left(V, m W^{\prime}\right) \rightarrow 0$ for $m \geqq c_{r-1}$, we get a decreasing sequence of nonnegative integers

$$
h^{1}\left(V, c_{r-1} W^{\prime}\right) \geqq h^{1}\left(V,\left(c_{r-1}+1\right) W^{\prime}\right) \geqq \cdots .
$$

Hence, for any pair $(V, W)$ in $\Sigma_{1}$, there are only two possibilities;

(1) $h^{1}\left(V,\left(c_{r-1}+b_{r}+j\right) W^{\prime}\right)=0$ for all $j \geqq 1$, or

(2) $h^{1}\left(V,\left(c_{r-1}+i\right) W^{\prime}\right)=h^{1}\left(V,\left(c_{r-1}+i+1\right) W^{\prime}\right)$ for some $i, 0 \leqq i \leqq b_{r}$.

Now we claim that (2) implies (1), i.e., (1) is always the case.

Suppose (2) is the case. Then, the following sequence is exact,

$$
0 \rightarrow H^{0}\left(V,\left(c_{r-1}+i\right) W^{\prime}\right) \rightarrow H^{0}\left(V,\left(c_{r-1}+i+1\right) W^{\prime}\right) \rightarrow H^{0}\left(W,\left(c_{r-1}+i+1\right) W^{\prime} \cdot W\right) \rightarrow 0 .
$$

This is equivalent to saying that $\operatorname{Tr}_{W}\left|\left(c_{r-1}+i+1\right) W^{\prime}\right|=\left|\left(c_{r-1}+i+1\right) W^{\prime} \cdot W\right|$. But,

$$
\begin{aligned}
\operatorname{Tr}_{W}\left|\left(c_{r-1}+i+2\right) W^{\prime}\right| & \supset \min \operatorname{sum}\left[\operatorname{Tr}_{W}\left(\phi^{-1}(|Z|)\right), \operatorname{Tr}_{W}\left|\left(c_{r-1}+i+1\right) W^{\prime}\right|\right] \\
& =\min \operatorname{sum}\left[\phi_{W}^{-1}(|Z|),\left|\left(c_{r-1}+i+1\right) W^{\prime} \cdot W\right|\right],
\end{aligned}
$$

and by $(r-1, B)$, the last linear system is complete and hence

$$
\left|\left(c_{r-1}+i+2\right) W^{\prime} \cdot W\right|=\operatorname{Tr}_{W}\left|\left(c_{r-1}+i+2\right) W^{\prime}\right| .
$$


Back in terms of exact sequences and $h^{1}$, we have

$$
h^{1}\left(V,\left(c_{r-1}+i+1\right) W^{\prime}\right)=h^{1}\left(V,\left(c_{r-1}+i+2\right) W^{\prime}\right),
$$

and repeating the same argument and using the semifineness argument, we get Case (1) as claimed.

Let $c_{r}=c_{r-1}+b_{r}$. Then, $(r, A)$ is true with the constant $c_{r}$. Now we claim that $(r, B)$ is also true with the same $c_{r}$. By $(r, A)$, we have $\operatorname{Tr}_{W}\left|m W^{\prime}\right|=\left|m W^{\prime} \cdot W\right|$ for $m \geqq c_{r}$ and from $c_{r} \geqq c_{r-1}$ and $(r-1, B)$ we have

$$
\min \operatorname{sum}\left[\operatorname{Tr}_{W}\left(\phi^{-1}(|Z|)\right), \operatorname{Tr}_{W}\left|m W^{\prime}\right|\right]=\left|(m+1) W^{\prime} \cdot W\right| \text { when } m \geqq c_{r} \text {. }
$$

We have to show

$$
\min \operatorname{sum}\left[\phi^{-1}(|Z|),\left|m W^{\prime}\right|\right]=\left|(m+1) W^{\prime}\right| \text { on } V \text { when } m \geqq c_{r} \text {. }
$$

Let the function module determined by $\phi^{-1}(|Z|)$ and a member $W^{\prime}$ be denoted by $L$ and the function module determined by min sum $\left[\phi^{-1}(|Z|),\left|m W^{\prime}\right|\right]$ and a member $(m+1) W^{\prime}$ be $M$ and the one determined by $\left|m W^{\prime}\right|$ and $m W^{\prime}$ be $L_{V}\left(m W^{\prime}\right)$, and so forth. Then, we have the following exact sequence of modules;

$$
\begin{gathered}
0 \rightarrow K \rightarrow M \\
\bigcap_{0 \rightarrow K^{\prime} \rightarrow L_{V}\left((m+1) W^{\prime}\right)} \rightarrow L_{W}\left((m+1) W^{\prime} \cdot W\right) \rightarrow 0
\end{gathered}
$$

where $K$ and $K^{\prime}$ are respective kernels of the map which sends functions on $V$ to their traces on $W$ (see [13] for terminology). It suffices to show $K^{\prime} \subset M$, i.e., $K=K^{\prime}$ and hence $\operatorname{dim} M=\operatorname{dim} L_{V}\left((m+1) W^{\prime}\right)$. Suppose $f \in K^{\prime}$, i.e., $(f)_{0}>W$. Then, $(f)=W+P-(m+1) W^{\prime}$ with some $P>0$, and hence $(f)=\left(W-W^{\prime}\right)$ $+\left(P-m W^{\prime}\right)$ gives $f=g \cdot h$ with $g \in L$ and $h \in L_{V}\left(m W^{\prime}\right)$, and hence $f \in M$.

To complete our proof, we need the following $\left(1, A^{*}\right),\left(1, B^{*}\right)$ and $c_{1}^{*}$ to replace $(r-1, A),(r-1, B)$ and $c_{r-1}$ for the case $r=2$.

$\left(1, A^{*}\right)$. Let $(V, C) \in \Sigma_{1}\left(V_{0}^{2}, C_{0}\right)$. On the nonsingular curve $C$, we use the RiemannRoch theorem for the $C$-divisor $m C^{\prime} \cdot C$, and get $H^{1}\left(C, m C^{\prime} \cdot C\right)=0$ for $m \geqq 2 g-2$, where the genus $g$ of the curve $C$ is a constant of the set $\Sigma_{1}$.

$\left(1, B^{*}\right) \cdot \operatorname{deg}\left(C^{\prime} \cdot C\right)=d>0$ is a constant of $\Sigma_{1}$ and hence we can find $c_{1}^{*}$ such that $c_{1}^{*} \geqq 2 g-2$ and for $m \geqq c_{1}^{*},(m-1) d \geqq 2 g-2$. Then, $\left|m C^{\prime} \cdot C\right|-C^{\prime} \cdot C$ is nonspecial and the Castelnuovo's lemma (Lemma 6 in [9]) becomes available. That is, $\min \operatorname{sum}\left[\phi_{c}^{-1}(|Z|),\left|m C^{\prime} \cdot C\right|\right]=\left|(m+1) C^{\prime} \cdot C\right|$ on $C$ for $m \geqq c_{1}^{*}$.

COROLlARY. Notations and assumptions being the same as above, there is a projective space $\boldsymbol{P}$ such that, for any pair $(V, W)$ in $\Sigma_{1}, V$ can be embedded into $\boldsymbol{P}$ by means of the complete linear system $\left|c_{r} W\right|$, and furthermore, the degree of the image variety is a constant of $\Sigma_{1}$.

Proof For any $(V, W)$ in $\Sigma_{1}, c_{r} W$ is an ample $V$-divisor because a very high multiple of $W$ is ample and then we can step downwards to $c_{r} W$ by $(r, B)$ and 
Lemma 5 of [9]. On the other hand, by $(r, A)$ and the constancy of $\chi\left(V, c_{r} W\right)$, $l\left(c_{r} W\right)$ is a constant of the set $\Sigma_{1}$. Hence, we can embed $V$ into $\boldsymbol{P}^{N}$ with $N+1=l\left(c_{r} W\right)$ by means of $\left|c_{r} W\right|$ for any $(V, W) \in \Sigma_{1}$. As the divisor $c_{r} W$ goes to a hyperplane section by the above embedding, and the degree of a zero cycle on $V$ obtained by intersecting $r$ members of $|W|$ being a constant of the set $\Sigma_{1}$, we get the second part of the corollary.

The above corollary comes very close to the notion of a universal subfamily mentioned in the introduction, and we put the definition down here formally.

Definition 7. Let $\Sigma$ be a subset of the set of deformations of a variety $\boldsymbol{V}_{0}$. A subset $\mathscr{U}$ of $\Sigma$ is called a universal subfamily of $\Sigma$ if the following conditions are satisfied:

(1) for any $V \in \Sigma$, there is a $V^{\prime} \in U$ such that $V \cong V^{\prime}$,

(2) there is a fixed projective space $\boldsymbol{P}$ such that $\boldsymbol{V}^{\prime} \subset \boldsymbol{P}$ for any $\boldsymbol{V}^{\prime} \in \mathscr{U}$,

(3) the set $\left\{c\left(V^{\prime}\right) \mid V^{\prime} \in U\right\}$ can be expressed as a finite union of open varieties, where $c\left(V^{\prime}\right)$ denotes the Chow-point of $V^{\prime}$.

2. $\alpha$-deformations and a universal subfamily. Let $\boldsymbol{V}$ be a variety with $\operatorname{dim} V \geqq 2$ such that an albanese $(\phi, A)$ of $V$ satisfies (1) $\operatorname{dim} V=\operatorname{dim} \phi(V)$ and (2) the morphism $\phi$ does not crush any curve on $V$ to a point. These properties do not depend on the choice of $(\phi, A)$ of $V$, and we will say that $V$ has property " $\alpha$ " if the above conditions are satisfied. For an $\alpha$-variety $V$ and a given albanese $(\phi, A)$ of $V$, we will construct a polarization $\mathscr{Y}$ on $A$ uniquely (and denote $A=(A, \mathscr{Y})$ ) and a polarization $\tilde{\mathscr{X}}$ on $V(\operatorname{denote} \tilde{V}=(V, \tilde{\mathscr{X}}))$ such that $(\tilde{V}, \phi)$ is properly $\Sigma(\boldsymbol{A})$-related.

Let us fix an $\alpha$-variety $V_{0}$ and an albanese $\left(\phi_{0}, A_{0}\right)$ of $V_{0}$. We define $\alpha$-deformation of $V_{0}$ by requiring each $V_{i}$ appearing in the chain of immediate deformations (in the Definition 2 of $\S 1$.) to be $\alpha$-varie ties. And $\Sigma_{\alpha}\left(V_{0}\right)$ will denote the set of $\alpha$-deformations of $V_{0}$. Then, comparing the two sets $\Sigma_{\alpha}\left(V_{0}\right)$ and $\Sigma\left(V_{0}, \phi_{0}\right)$, we will construct a universal subfamily of $\Sigma_{\alpha}\left(V_{0}\right)$ in this section.

Let $V$ be an $\alpha$-variety and $X$ be an ample polar divisor of $V$. Let $C$ be a 1-cycle of the form $X_{1} \cdot X_{2} \cdots \cdot X_{r-1}$ where $X_{i} \in|X|$ such that the intersection is defined. Let $\left(\phi, A^{q}\right)$ be an albanese of $V$. First, we know that, by suitable choice of $C$, $\phi(C)$ generates $A$ and the Pontrjagin sum of $\phi(C), q-1$ times in $A$, gives a nondegenerate $A$-divisor $Y[5]$.

Lemma 1. Let $V$ and $(\phi, A)$ be as before, and $D_{i}(i=1,2)$ be 1-cycles on $V$ whose supports are irreducible curves $C_{i}$ respectively. Suppose $D_{1} \equiv D_{2}$ (alg. eq.). Then, $\operatorname{pr}_{2}\left[\left(D_{1} \times A\right) \cdot \Gamma_{\phi}\right]$ and $\operatorname{pr}_{2}\left[\left(D_{2} \times A\right) \cdot \Gamma_{\phi}\right]$ are numerically equivalent to each other on $A$.

Proof. For any $A$-divisor $W$ such that

$$
\left\{\operatorname{pr}_{2}\left[\left(D_{1} \times A\right) \cdot \Gamma_{\phi}\right]-\operatorname{pr}_{2}\left[\left(D_{2} \times A\right) \cdot \Gamma_{\phi}\right]\right\} \cdot W
$$

is defined, we want $\left.\operatorname{deg}\left\{\operatorname{pr}_{2}\left[\left(D_{1} \times A\right) \cdot \Gamma_{\phi}\right]-\operatorname{pr}_{2}\left[\left(D_{2} \times A\right) \cdot \Gamma_{\phi}\right]\right\} \cdot W\right)=0$. By our 
assumption and changing $W$ within the algebraic equivalence class (hence within the numerical equivalence class) if necessary, we may assume $\left(\operatorname{pr}_{2}\left(D_{i} \times A\right) \cdot \Gamma_{\phi}\right) \cdot W$ to be defined for each $i=1,2$. Then by the property $\alpha$ of $\phi, E_{t} \cdot(V \times W)$ are defined on $\Gamma_{\phi}$, where $E_{1}, E_{2}$ are transforms of $D_{1}, D_{2}$ respectively by the isomorphism between $V$ and $\Gamma_{\phi}$. As the algebraic projection preserves the degree of 0 -cycles, we get the lemma.

Lemma 2. Let $V$ and $(\phi, A)$ be as before. Then, there is a polarization $\mathscr{Y}$ on $A$ which is uniquely determined by $V$ and $\phi$.

Proof. By the remark before Lemma 1 , there is a polarization on $A$ determined by $Y$. Suppose now that $X_{1}$ is another ample polar divisor of $V$. Then, there are integers $a$ and $a_{1}$ such that $a X \equiv a_{1} X_{1}$ (alg. eq.) and if $C_{1}$ is a curve obtained from $X_{1}$ in the same way as we got $C$ from $X$, then $a^{r-1} C \equiv a_{1}^{r-1} C_{1}$ (alg. eq.). Then, by Lemma 1 and by linearly extending the Pontrjagin sum, we see that some multiple of $Y$ is numerically equivalent to some multiple of $Y_{1}$ and we get the same polarization on $A$.

By this lemma, we call $(\phi, \boldsymbol{A})$ an albanese of $\boldsymbol{V}$, where $\boldsymbol{A}=(\boldsymbol{A}, \mathscr{Y}), \mathscr{Y}$ being the polarization determined by $Y$ in the above notation.

LEMMA 3. With the notations as above, $\boldsymbol{A}$ gives a new polarization $\mathscr{X}(A)$ on the underlying variety $V$ of $V$. If we denote $V(A)=(V, \mathscr{X}(A))$, then $\phi: V(A) \rightarrow \boldsymbol{A}$ is a morphism. Furthermore, if $(\psi, B)$ were another albanese of $V$, then $\mathscr{X}(A)=\mathscr{X}(B)$. (Hence, we denote $\tilde{V}=(V, \mathscr{X}(A))$.)

Proof. Let $Y$ be an ample polar divisor of $A$. Then, we can find $Y_{1}$, linearly equivalent to $Y$, such that $\operatorname{pr}_{1}\left[\Gamma_{\phi} \cdot\left(V \times Y_{1}\right)\right]=\phi^{-1}\left(Y_{1}\right)$ is defined. Then, by the property $\alpha, \phi^{-1}\left(Y_{1}\right)$ is nondegenerate on $V$ and hence gives $\mathscr{X}(A)$. The last part of the lemma comes from the universal mapping property of an albanese map.

With the notations of Lemma $3,(\tilde{V}, \phi)$ is properly $\Sigma(A)$-related and for any other albanese $\left(\phi^{\prime}, A^{\prime}\right)$ of $V$, we have $(\tilde{V}, \phi) \cong\left(\tilde{V}, \phi^{\prime}\right)$ in the sense of Definition 4, §1.

Now let us consider specializations. Let $V$ be an $\alpha$-variety and fix an albanese $(\phi, A)$ of $V$. Then, we have $\boldsymbol{A}$ and $\tilde{V}$ uniquely by Lemma 2 and Lemma 3 . Let $V_{1}$ be a nonsingular variety such that $V \sim \rightarrow V_{1}$. Then, for an albanese $\left(\phi_{1}, A_{1}\right)$ of $V_{1}, \operatorname{dim} A=\operatorname{dim} A_{1}=q$. On the other hand, there is an albanese $\left(\phi^{\prime}, A^{\prime}\right)$ of $V$ such that $\left(V, \Gamma_{\phi^{\prime}}, A^{\prime}\right) \sim \rightarrow\left(V_{1}, \Gamma_{\psi}, B\right)$ with the property that $B$ is abelian and $\left(\psi, V_{1}\right)$ generates $B$. By the behavior of an abelian subvariety over the specialization of an abelian variety, and the fact that we are in characteristic 0 , $(\psi, B)$ is actually an albanese of $V_{1}[3],[4]$.

LEMMA 4. Let $V$ be an $\alpha$-variety and fix an albanese $(\phi, A)$ of $V . V_{1}$ be another $\alpha$-variety such that $V \backsim \rightarrow V_{1}$. Then, for any albanese $\left(\phi_{1}, A_{1}\right)$ of $V_{1}$, we have $(\tilde{V}, \phi) n \rightarrow\left(\tilde{V}_{1}, \phi_{1}\right)$. 
Proof. From $V \backsim \rightarrow V_{1}$, we have $\left(V, \Gamma_{\phi^{\prime}}, A^{\prime}\right) \backsim \rightarrow\left(V_{1}, \Gamma_{\psi}, B\right)$ with

$$
(\tilde{V}, \phi) \cong\left(\tilde{V}, \phi^{\prime}\right) \text { and }\left(\tilde{V}_{1}, \phi_{1}\right) \cong\left(\tilde{V}_{1}, \psi\right) \text {. }
$$

We can choose ample polar divisors $X$ and $X_{1}$ respectively of $V$ and $V_{1}$ such that $\left(V, \Gamma_{\phi^{\prime}}, A^{\prime}, X\right) \sim \rightarrow\left(V_{1}, \Gamma_{\psi}, B, X_{1}\right)$. In the construction of the nondegenerate divisors $Y$ and $Y_{1}$ respectively of $A^{\prime}$ and $B$ (from $X$ and $X_{1}$, in Lemma 2), we used operations which are compatible with specialization. Hence, $\left(V, \Gamma_{\phi^{\prime}}, A^{\prime}\right)$ $n \rightarrow\left(V_{1}, \Gamma_{\psi}, B\right)$. Now, we can choose ample polar divisors $Y^{\prime}$ and $Y_{1}^{\prime}$ of $A^{\prime}$ and $B$ respectively so that $Y^{\prime} \backsim \rightarrow Y_{1}^{\prime}$ over the already constructed specialization. Then, the operations involved in obtaining $\tilde{\mathscr{X}}$ and $\tilde{\mathscr{X}}_{1}$ from $Y^{\prime}$ and $Y_{1}^{\prime}$ respectively (in Lemma 3$)$ are again compatible with specialization. i.e., $\left(\tilde{V}, \Gamma_{\phi^{\prime}}, A^{\prime}\right) \backsim \rightarrow\left(\tilde{V}_{1}, \Gamma_{\psi}, B\right)$ over $V \backsim \rightarrow V_{1}$.

Let us fix an $\alpha$-variety $V_{0}$ and an albanese $\left(\phi_{0}, A_{0}\right)$ of $V_{0}$. And consider the following two sets: $\Sigma_{\alpha}\left(V_{0}\right)$ and $\Sigma\left(\tilde{V}_{0}, \phi_{0}\right)$. For any nember $V$ of $\Sigma_{\alpha}$ and any albanese $(\phi, A)$ of $\left.V,(\tilde{V}, \phi) \in \Sigma \tilde{V}_{0}, \phi_{0}\right)$. Applying the Corollary of Proposition 1, $\S 1$, to the subset of pairs $(V, W)$ in $\Sigma_{1}\left(V_{0}, W_{0}\right)$ which arise from $(\tilde{V}, \phi) \in \Sigma\left(\tilde{V}_{0}, \phi_{0}\right)$ such that $V \in \Sigma_{\alpha}$ and $\phi$ is an albanese map of $V$, we have:

PRoposition 2. Underlying varieties of members of $\Sigma_{\alpha}\left(V_{0}\right)$ can be embedded into a fixed projective space so that the degree of the image variety is a constant of the set $\Sigma_{\alpha}$.

THEOREM 1. Let $V_{0}$ be an $\alpha$-variety. The set of $\alpha$-deformations of $V_{0}, \Sigma_{\alpha}\left(V_{0}\right)$, considered as a subset of $\Sigma\left(V_{0}\right)$, has a universal subfamily.

Froof. Let $\boldsymbol{P}$ be the projective space appearing in Proposition 2 above and $\mathscr{U}$ be the subset of $\Sigma_{\alpha}$, consisting of embedded images in $\boldsymbol{P}$ with the transferred (by embedding) polarizations. For any $U \in \mathscr{U}$, a pair $(U, B)$, where $B$ is a basic polar divizor of $\boldsymbol{U}$, has the following property: $\left|B+C_{m}\right|$ on $U$ is not empty where $m$ is a suitable constant of the set $\mathscr{U}$ and $C_{m}$ denotes an $m$-ple of hyperplane section of $U$. $\left(\left|B+C_{m}\right|\right.$ becomes ample if $m \geqq m_{0}$ where $m_{0}$ depends on the degree of $B$ which is a constant of the set $\mathscr{U}$.) Hence, the iunderlying varieties of members of $\mathscr{U}$ from a subset of the set of nonsingular varieties in $\boldsymbol{P}$ of a fixed degree $d(=\operatorname{deg}(U))$ which carry at least a positive divisor of a fixed degree $e\left(=\operatorname{deg}(Z)\right.$ with $\left.Z \in\left|B+C_{m}\right|\right)$.

Let the Chow-bunch of positive cycles in $\boldsymbol{P}$ of dimension $r$, degree $d$, be $\mathscr{A}$ and that of dimension $r-1$, degree $e$, be $\mathscr{B}$, and fix a pair $\left(U_{0}, Z_{0}\right)$ where $U_{0}$ is an underlying variety of a member of $\mathscr{U}$ and $Z_{0}$ is a positive $U_{0}$-cycle in $\left|B_{0}+C_{m}\right|$. Choose an irreducible component $\mathscr{A}_{0}$ of $\mathscr{A}$ containing $c\left(U_{0}\right)$, and an irreducible component $\mathscr{B}_{0}$ of $\mathscr{B}$ containing $c\left(Z_{0}\right)$. The possible choices of the pair $\left(\mathscr{A}_{0}, \mathscr{B}_{0}\right)$ are finite in number. From the graph of the algebraic correspondence between $\mathscr{A}_{0}$ and $\mathscr{B}_{0}$, which associates $c(U)$ to $c(Z)$ so that the support of $U$ contains the support of $Z$, choose an irreducible component $\mathscr{C}_{0}$ which contains the point $\left(c\left(U_{0}\right), c\left(Z_{0}\right)\right)$. (The set of such components is nonempty, finite.) 
Let $(\bar{u}, \bar{z})$ be a generic point of $\mathscr{C}_{0}$ and $\bar{U}$ and $Z$ be $P$-cycles so that $c(\bar{U})=\bar{u}$, $c(Z)=\bar{z}$, respectively. Then, from $(\bar{U}, Z) \sim \rightarrow\left(U_{0}, Z_{0}\right)$ we can deduce the following:

(a) $\bar{U}$ is a nonsingular variety which has the property $\alpha$, and

(b) let $\bar{B}=Z-\bar{C}_{m}$ where $\bar{C}_{m}$ is an $m$-ple of a hyperplane section of $\bar{U}$. Then, $\bar{B}$ is a nondegenerate $\bar{U}$-divisor and the polarization determined by $\bar{B}$ on $\bar{U}$ gives a variety $\bar{U}$ so that $\vec{U} \sim \rightarrow U_{0}\left(U_{0} \in \mathscr{U}\right)$.

Let the geometric projection of $\mathscr{C}_{0}$ into $\mathscr{A}_{0}$ be $\mathscr{C}_{0}^{\prime}$, and denote by $\mathscr{C}_{0}^{\prime \prime}$ the subset of points $c(U)$ so that $U$ is nonsingular and has property $\alpha$. Then, $\mathscr{C}_{0}^{\prime \prime}$ is an open subvariety of $\mathscr{C}_{0}^{\prime}$. In the process of obtaining $\mathscr{C}_{0}^{\prime \prime}$, we were given only finite choices in each step. Let $\mathscr{C}^{\prime \prime}$ be the set union of all possible $\mathscr{C}_{0}^{\prime \prime}$. Then, $\mathscr{C}^{\prime \prime}$ has the following property: (a) if $U \in \mathscr{U}$, then $c(U) \in \mathscr{C}^{\prime \prime}$, and (b) for any $c(U) \in \mathscr{C}^{\prime \prime}$, we can give a polarization on $U$, in a uniform manner, so that $U \in \Sigma_{\alpha}$. From (b) we obtain a subset $\mathscr{U}^{\prime \prime}$ of $\Sigma_{\alpha}$ so that $\mathscr{U} \subset \mathscr{U}^{\prime \prime}$ by (a), and hence $\mathscr{U}^{\prime \prime}$ is a universal subfamily of $\Sigma_{\alpha}$.

\section{REFERENCES}

1. H. Hironaka, A note on algebraic geometry on ground rings, Illinois J. Math. 2 (1958), 355-366.

2. W. Hoyt, On products and algrbraic families of Jacobian varieties, Ann. of Math. 77 (1963), 415-423.

3. S. Koizumi, On specialization of the Albanese and Picard varieties, Mem. Coll. Sci. Univ. Kyoto Ser. A Math. 32 (1960), 371-382.

4. S. Koizumi and G. Shimura, On specializations of abelian varieties, Sci. Papers Coll. Gen. Ed. Univ. Tokyo 9 (1959), 187-211.

5. S. Lang, Abelian varieties. Interscience Tracts in Pure and Appl. Math, No. 7, Interscience, New York, 1959.

6. Y. Nakai, A criterion of an ample sheaf on a projective scheme, Amer. J. Math. 135 (1963), $14-26$.

7. T. Matsusaka, On algebraic families of positive divisors and their associated varieties on a projective variety, J. Math. Soc. Japan 5 (1953), 113-135.

8. - Polarized varieties, field of moduli and generalized Kummer varieties of polarized abelian varieties, Amer. J. Math. 80 (1958), 45-82.

9. T. Matsusaka and D. Mumford, Two fundamental theorems on deformations of polarized varieties, Amer. J. Math. 136 (1964), 668-684.

10. G. Shimura, Modules des varietes abeliennes polarisees et fonctions modulaires, Fonctions Automorphes, Séminaire Henri Cartan, Ann. Sci. École Norm. Sup. 2 (1957-1958), 18-01-20-18.

11. A. Weil, Foundations of algebraic geometry, Amer. Math. Soc. Colloq. Publ. Vol. 29 Amer. Math. Soc., Providence, R. I., 1962.

12. O. Zariski, Complete lirear systems on normal varieties and a generalization of a lemma of Enriques-Serveri, Ann. of Math. 55 (1952), 552-592.

13. - Several complex variables, Part III, Algebraic sheaf theory (Scientific Report on the Second Summer Institute), Bull. Amer. Math. Soc. 62 (1956), 117-141.

PURDUE UNIVERSITY,

LAFAYETTE, INDIANA 